experienced symptoms and distress (Scobie, 2021). There has been very limited examination of palliative and end-of-life care in care homes during COVID-19, or strategies to improve this.

Aims To examine the experiences of care homes in England of providing palliative and end-of-life care during the COVID19 pandemic and make recommendations for policy.

Methods Online survey (in REDCap) of care home staff with leadership responsibilities, identified through established networks. The survey included structured data and free-text comments on COVID-19 outbreaks, experiences of symptom assessment and control, and impact on workforce. The primary outcome was staff self-efficacy to provide palliative and end-of-life care (Phillips, Salamonson, Davidson, 2011).

Results Interim analysis of 66 respondents found that most staff felt confident to provide palliative care, as measured by the self-efficacy scale (median 3.75, range 1-4 ). 51\% (33/65) of respondents identified issues with staff shortages during the pandemic; 38\% (24/64) experienced changes in staff responsibilities; 18\% (12/65) experienced challenges in recognising that residents may be dying. 18\% (12/65) of care homes did not allow visitors at the end-of-life, and 39\% (25/64) experienced difficulties in accessing help from other services. 51\% $(33 / 65)$ experienced challenges in providing bereavement support to relatives. Free-text comments identified staff shortages and exhaustion, and lack of support from other healthcare services, as barriers to good care.

Conclusion This is the first national survey to explore provision of palliative and end-of-life care in care homes during the COVID-19 pandemic. The results will be used to inform policy to ensure high-quality provision of palliative and endof-life care during future pandemics.

\section{P-199 ABOUT YOU, FOR YOU, WITH YOU - SUPPORTING CARE HOMES THROUGHOUT A PANDEMIC}

Fiona Irvine. Ayrshire Hospice, Ayr, UK

\subsection{6/spcare-2021-Hospice.215}

Background In response to the COVID-19 pandemic Ayrshire Hospice established a ten-week, live online supportive education programme for Care Homes using Project Echo ${ }^{\circledR}$. Project Echo ${ }^{\circledR}$ is a widely used model for education, aiming to improve care by gathering a community of practice together for learning and support with the goal of improving decision making by collaborative problem solving'.

Local care homes were asked what educational support they needed, and the hospice responded, using the multidisciplinary team, to specific topic requests. This gave participants an opportunity to build links, increase knowledge, share practices and experiences through supportive facilitation.

Aims To use the hospice team and Project Echo ${ }^{\circledR}$ to connect with and support care home staff dealing with the unprecedented challenges of providing palliative and end of life care during COVID-19.

Methods

- Core facilitation team established.

- Liaison with Highland and Accord hospices regarding their Project Echo ${ }^{\circledR}$ experience.

- Establish links with AAHB and HSCP to determine existing practice, need, create awareness.

- Developed systems required to support the programme.
- Contacted every care home across Ayrshire to determine interest and need

- Evaluation developed.

\section{Outcomes}

- Responding to COVID-19, an intended service development was quickly established and implemented, providing access to specialist palliative care/advice and support to care homes.

- Identified learning needs were met, with weekly agendas set by care homes.

- Safe space to share knowledge and experience without needing to travel

- Peer support.

- Established/improved links with the hospice/AAHB/HSCP and care homes across Ayrshire.

- Evaluated extremely well - evidence of shared learning, improved care, links established and a sense of support and understanding.

- Aligned with the NHS Scotland' Palliative and end of life framework.

- Future plans - Project Echo ${ }^{\circledR}$ to be used as educational model, to improve palliative and end-of-life care in Ayrshire by increasing capacity and capability of community teams.

\section{P-200 USING PROJECT ECHO TO PROVIDE EDUCATION, ADVICE AND SUPPORT TO GENERAL PRACTITIONERS}

${ }^{1}$ Janet Diffin, ${ }^{1}$ Tracey McTernaghan, ${ }^{2}$ Aine McMullan, ${ }^{2}$ Martin Hayes, ${ }^{1}$ Max Watson, ${ }^{1}$ Christopher Jenkins. ${ }^{1}$ Hospice UK, Belfast, UK: ${ }^{2}$ Health and Social Care Board, Belfast, UK

\subsection{6/spcare-2021-Hospice.216}

Introduction Project ECHO (Extension for Community Healthcare Outcomes) is a tele-mentoring programme that uses video-conferencing technology to deliver, evidencebased education from specialists and case-based learning with peers.

The Health and Social Care Board in Northern Ireland identified Project ECHO as a methodology to implement change. The vision was to engage with medical professionals to develop communities of practice, enhance knowledge, skills, and ultimately improve patient/client care. Project ECHO networks were established with a specific focus on (neurology, gynaecology, musculoskeletal, dermatology, ENT, cardiology, diabetes, and gastroenterology), along with a 'GP support' network. Network participants identified topics to be included within their curriculum ensuring a tailored approach to education delivery. Participants set the network objectives and the outcomes they hoped to achieve. ECHO sessions were held monthly and each network typically delivered nine sessions.

Method Participatory monitoring and evaluation was used to identify outcomes for ECHO network participants. Evaluation surveys were issued after the final session.

Results 361 medical professionals attended at least one ECHO session across the networks outlined above. 36\% (131/361) responded to the evaluation survey. Results indicated participants valued access to education from specialists, to ask questions directly, and seek advice from secondary care colleagues. Participants reported knowledge of conditions and symptoms, confidence and capacity to treat patients in primary care increased. Improvements in relationships between primary and secondary care were also reported. Benefits reported by 'GP 